\title{
Initial Seizure Threshold in Brief-Pulse Bilateral Electroconvulsive Therapy in Patients with Schizophrenia or Schizoaffective Disorder
}

\author{
Seong Hoon Jeong', Tak Youn 2,3 , Younsuk Lee ${ }^{4}$, Jin Hyeok Jang ${ }^{2}$, \\ Young Wook Jeong ${ }^{2}$, Yong Sik Kim², ${ }^{2,3}$ and In Won Chung ${ }^{2,3} \bowtie$ \\ ${ }^{1}$ Department of Psychiatry, Eulji University Hospital, Daejeon, Republic of Korea \\ 2Department of Psychiatry and Electroconvulsive Therapy Center, Dongguk University International Hospital, Goyang, Republic of Korea \\ ${ }^{3}$ Institute of Clinical Psychopharmacology, Dongguk University College of Medicine, Goyang, Republic of Korea \\ ${ }^{4}$ Department of Anesthesiology and Pain Medicine, Dongguk University International Hospital, Goyang, Republic of Korea
}

Objective The present study aimed to report the initial seizure threshold (IST) of a brief-pulse bilateral electroconvulsive therapy (BPBL ECT) in Korean patients with schizophrenia/schizoaffective disorder and to identify IST predictors.

Methods Among 67 patients who received ECT and diagnosed with schizophrenia/schizoaffective disorder based on the criteria of the Diagnostic and Statistical Manual of Mental Disorders, Fourth Edition, Text Revision, we included 56 patients who received 1-millisecond BP-BL ECT after anesthesia with sodium thiopental between March 2012 and June 2018. Demographic and clinical information was gathered from electronic medical records, and a multiple regression analysis was conducted to identify predictors of the IST.

Results The mean age of the patients was $36.9 \pm 12.0$ years and $30(53.6 \%)$ patients were male. The mean and median IST were $105.9 \pm 54.5$ and 96 millicoulombs $(\mathrm{mC})$, respectively. The IST was predicted by age, gender, and dose $(\mathrm{mg} / \mathrm{kg})$ of sodium thiopental. Other physical and clinical variables were not associated with the IST.

Conclusion The present study demonstrated that the IST of 1-ms BP-BL ECT following sodium thiopental anesthesia in Korean patients was comparable to those reported in previous literature. The IST was associated with age, gender, and dose of sodium thiopental.

Psychiatry Investig 2019;16(9):704-712

Key Words Schizophrenia, Schizoaffective disorder, Initial seizure threshold, Electroconvulsive therapy, Brief-pulse width, Bilateral electrode placement.

\section{INTRODUCTION}

Electroconvulsive therapy (ECT) is a safe and effective treatment for various psychiatric disorders and conditions. ${ }^{1,2}$ The successful induction of a generalized seizure requires an adequate dose of electricity that exceeds the seizure threshold, which is defined as the minimal electrical intensity necessary to produce a seizure. ${ }^{3,4}$ The initial seizure threshold (IST) determined at the first ECT session guides the electrical doses for

\footnotetext{
Received: March 3, 2019 Revised: April 16, 2019

Accepted: June 20, 2019

$\triangle$ Correspondence: In Won Chung, $\mathrm{MD}, \mathrm{PhD}$

Department of Psychiatry and Electroconvulsive Therapy Center, Dongguk University International Hospital, Institute of Clinical Psychopharmacology, Dongguk University School of Medicine, 27 Dongguk-ro, Ilsandong-gu, Goyang 10326, Republic of Korea

Tel: +82-31-961-7231, Fax: +82-31-961-7236, E-mail: ciwkjs@gmail.com

(c) This is an Open Access article distributed under the terms of the Creative Commons Attribution Non-Commercial License (https://creativecommons.org/licenses/bync/4.0) which permits unrestricted non-commercial use, distribution, and reproduction in any medium, provided the original work is properly cited.
}

the entire following sessions, and thus, considered to be one of the most important parameters of ECT procedure. ${ }^{5}$ Because unnecessary exposure to higher electrical doses produces more severe cognitive impairment, ${ }^{6-8}$ determining IST is particularly important for ECT optimization with better clinical efficacy and minimal cognitive side effect., ${ }^{910}$

However, IST determination is not a straightforward task, as it varies up to 40 times for different individuals. ${ }^{11,12}$ IST is known to be associated with patients' own characteristics (age, sex, body mass index, etc.) and/or treatment factors (ECT parameters, anesthetics, concomitant medication, etc.). ${ }^{3,13-17}$ For example, the IST is higher in older age, ${ }^{3,13,16}$ in male, ${ }^{3,13,16}$ in bilateral electrode placement, ${ }^{14,15,17,18}$ and following the use of certain anesthetic agents. ${ }^{19}$ On the other hand, the effects of severity or duration of illness, presence of psychosis, or the use of concomitant medications remain controversial or largely irrelevant. ${ }^{10,20-24}$

Several methods of IST determination have been proposed, 
including age-based, half-age-based, fixed-high, and empirical titration methods. ${ }^{4,825,26}$ These methods can be categorized into two approaches: 1) the administration of a predetermined fixed-dose modified by the above variables and 2) the titration by repeated trials in which the dose is incrementally raised until a satisfactory seizure appears. However, controversy remains regarding which of these is the most desirable method. ${ }^{27}$

The influence of race and ethnicity on IST is not yet clear. ${ }^{21}$ Most studies on IST had been conducted in Western populations and only a handful of studies reported IST values in Asian populations. ${ }^{23,24,28-30}$ For example, Yasuda et al..$^{23}$ assessed IST in Japanese patients (age range of 20-83 years) using 0.5-ms pulse with Thymatron SYSTEM IV ECT apparatus. They found that the IST was equal or slightly higher than those reported in other ethnic groups. In contrast, Lee et al. ${ }^{31}$ reported that the IST of Korean patients (age range of 20-73 years) using 1.4-ms pulse with MECTA SR-1 model was lower than that of Western populations. These findings were not directly comparable to each other due to differences in the used pulse width, ECT devices, titration schedules, and age distributions. However, considering ethnicity may still be important in estimating IST in actual clinical practice. ${ }^{23,32,33}$

The present retrospective study based on chart-review aimed to report the distribution of IST values in Korean patients following a specific protocol. Especially, this study had focused on patients diagnosed with either schizophrenia or schizoaffective disorder. To date, studies on IST done with psychotic patients were hard to find. ${ }^{28,34}$ However, schizophrenia and other psychotic illnesses are another important indication for ECT, especially in treatment-resistant groups. ${ }^{35-37}$ The protocol used in this study required that all the study participants received 1-millisecond (ms) brief-pulse bilateral (BP-BL) ECT following general anesthesia with sodium thiopental. The IST was empirically titrated by incrementally increasing the stimulus intensity, according to a predefined schedule. Additionally, the present study evaluated the association of IST with various demographic and clinical characteristics to identify factors that may be predictive of IST. A multiple regression model was built using those characteristics as potential predictors. The obtained results would provide valuable information in conducting ECT in patients with schizophrenia and schizoaffective disorder, especially in patients whose physical condition does not allow empirical titration procedure. ${ }^{38,39}$

\section{METHODS}

\section{Subjects}

Patients who underwent an acute course of ECT under the diagnosis of schizophrenia or schizoaffective disorder from March 2012 to June 2018 at the ECT center of Dongguk Uni- versity International Hospital were identified as eligible subjects. Diagnoses were confirmed by the physician-in-charge and one of the authors (CIW) after the chart review in accordance with the Diagnostic and Statistical Manual of Mental Disorders, Fourth Edition, Text Revision (DSM-IV-TR).

In an effort to report the IST distribution following a specific protocol, only the patients who had received 1-ms BP-BL ECT after anesthesia with sodium thiopental were selected, which constituted the majority of the all eligible subjects. Information on demographic characteristics, clinical characteristics, ECT history, and use of concomitant medications (antipsychotics, anticonvulsants, antidepressants, and benzodiazepines) was obtained from electronic medical records (EMR). The study proposal was approved by the Institutional Review Board of Dongguk University International Hospital (2018-10-003).

\section{ECT procedure}

The ECT session was commenced only after completion of psychiatric and medical work-ups. The administration of anticonvulsants and/or benzodiazepines was stopped or reduced the day before the ECT session in all patients. The MECTA spECTrum 5,000Q (MECTA Corp; Lake Oswego, OR, USA) was used to deliver the electrical stimulus, and BP-BL ECT was the primary recommendation if there were no concerns about memory or cognitive impairments. The seizure was monitored in three ways: electroencephalography (EEG), electrocardiography (ECG), and motor movement measuring by an optical motion sensor (OMS) under the tourniquet method. The anesthesia and muscle relaxation procedures were described in detail in a previous report issued by our research group. ${ }^{37}$ All patients were continuously monitored until full recovery of consciousness and the return of stable vital signs.

\section{IST measurement}

The IST was measured at the first ECT session by empirical titration method. The stimulus intensity was increased according to a predetermined schedule (Table 1), which had been modified from the recommended dose table by Coffey et al., ${ }^{40}$ and those of Seoul National University Hospital and University of Pittsburgh Medical Center (UPMC). ${ }^{37}$ The electrical dose was increased from the lowest level up to five times until an adequate seizure appeared. Adequate seizure duration was considered to be $\geq 25 \mathrm{sec}$ according to EEG or $\geq 20$ sec according to OMS, and seizure quality was evaluated by three EEG patterns of polyspike activity, three spikes and wave activity, and post-ictal suppression. ${ }^{41}$

\section{Statistical analysis}

The demographic and clinical characteristics of the patients are presented in Table 1 . To compare genders, continuous vari- 
ables were assessed with Student t-tests, and categorical variables were assessed with chi-square tests. A multiple regression analysis was conducted to identify the relevant predictors of IST; the candidate predictors included demographic variables (age, gender, duration of illness, family history of psychiatric illness), physical variables (weight, height, and BMI), ECT procedure variables (anesthetic agent dose) and variables related to medication use (use of clozapine/anticonvulsants/ antidepressants/benzodiazepines, and chlorpromazine-equivalent doses of antipsychotic medication). A stepwise variable selection procedure was employed to identify the most parsimonious set of relevant predictors. The finding strategy was set to "both directions", and the criteria for the best model were Akaike's Information Criteria (AIC). After the best model was obtained, more complicated models were built in consideration of the possible interactions among the identified predictors. The significance levels of the interactions were determined based on the F-test values obtained in comparisons between the more complicated models and the base model. The $\mathrm{p}$-values of less than 0.05 were considered to indicate statistical significance. All statistical analyses were performed using SPSS ver. 23.0 for Windows (IBM Corp, Armonk, NY, USA) and an open-source statistical program R (ver. 3.2.4: R Project for Statistical Computing, Vienna, Austria). ${ }^{42}$

\section{RESULTS}

\section{Demographic and clinical characteristics}

In total, 67 patients with schizophrenia or schizoaffective disorder received ECT during the study period. Among them, 56 (83.5\%) had undergone a 1-ms BP-BL ECT after anesthesia with sodium thiopental. These patients were included in the present analysis. The excluded patients were 1) seven patients with the right unilateral electrode placement and 2) four patients with propofol-induced anesthesia.

The mean age of the study subjects was $36.9 \pm 12.0$ years, and 30 patients $(53.6 \%)$ were male. There were no gender differences in educational level, occupation, family history, intelligence quotient (IQ) proportion and duration of illness, but the mean BMI of the male subjects $(27.1 \pm 5.4)$ was significantly higher than that of the female subjects $(24.0 \pm 5.3 ; \mathrm{t}=2.158, \mathrm{p}=$ $0.035)$. The medication-related variables, such as chlorpromazine-equivalent dose, use of clozapine, and use of concomitant anticonvulsants, antidepressants, or benzodiazepines, did not differ between the male and female subjects (Table 1).

\section{Distribution of IST values}

The mean IST of the study subjects was $105.9 \pm 54.5$ millicoulomb (mC; range: 48-288 $\mathrm{mC}$ ) (Table 1). The distribution was right-skewed with a mean value greater than median val-

Table 1. Summary of demographic, ECT, medication related variables and the estimated IST of the study subjects who underwent BP-BL ECT after anesthesia with sodium thiopental $(\mathrm{N}=56)$

\begin{tabular}{|c|c|c|c|c|}
\hline Variables & Total $(\mathrm{N}=56)$ & Male $(\mathrm{N}=30)$ & Female $(\mathrm{N}=26)$ & $\begin{array}{c}\text { Male vs. female } \\
\text { p-values }\end{array}$ \\
\hline \multicolumn{5}{|l|}{ Demographic variables } \\
\hline Age (years) & $36.9 \pm 12.0^{*}$ & $33.4 \pm 11.5$ & $40.9 \pm 11.5$ & 0.018 \\
\hline Weight (kg) & $70.6 \pm 18.1$ & $79.7 \pm 16.0$ & $60.2 \pm 14.5$ & $<0.001$ \\
\hline Height $(\mathrm{cm})$ & $165.5 \pm 8.5$ & $171.6 \pm 4.9$ & $158.4 \pm 5.8$ & $<0.001$ \\
\hline $\mathrm{BMI}\left(\mathrm{kg} / \mathrm{m}^{2}\right)$ & $25.6 \pm 5.5$ & $27.1 \pm 5.4$ & $24.0 \pm 5.3$ & 0.035 \\
\hline Duration of illness (years) & $12.2 \pm 7.8$ & $12.5 \pm 7.9$ & $11.8 \pm 7.8$ & 0.729 \\
\hline \multicolumn{5}{|l|}{ ECT variables } \\
\hline Thiopental dose (mg) & $214.7 \pm 62.5$ & $240.8 \pm 59.3$ & $184.6 \pm 52.5$ & $<0.001$ \\
\hline Thiopental dose/weight (mg/kg) & $3.1 \pm 0.6$ & $3.0 \pm 0.5$ & $3.1 \pm 0.7$ & 0.725 \\
\hline IST (millicoulomb) & $105.9 \pm 54.5$ & $115.2 \pm 59.9$ & $95.2 \pm 46.3$ & 0.174 \\
\hline \multicolumn{5}{|l|}{ Concomitant medications } \\
\hline Chlorpromazine-equivalent dose of antipsychotics (mg) & $667.2 \pm 458.6$ & $695.6 \pm 430.8$ & $634.4 \pm 495.3$ & 0.623 \\
\hline Clozapine use (yes/no) & $40 / 16$ & $20 / 10$ & $20 / 6$ & 0.102 \\
\hline Anticonvulsant use (yes/no) & $27 / 29$ & $16 / 14$ & $11 / 15$ & 0.579 \\
\hline Antidepressant use (yes/no) & $14 / 42$ & $8 / 22$ & $6 / 20$ & 1.000 \\
\hline Benzodiazepine use (yes/no) & $39 / 17$ & $16 / 10$ & $23 / 7$ & 0.349 \\
\hline
\end{tabular}

*numbers are expressed as mean \pm standard deviation or as the frequency of yes/no. The difference between male and female subjects was statistically tested with Student t-tests for continuous variables and chi-square test for dichotomous variables. ECT: electroconvulsive therapy, IST: initial seizure threshold, BP-BL: Brief-pulse Bilateral, BMI: body mass index 
ue $(96 \mathrm{mC})$ (Figure 1). The mean IST of the males (115.2 \pm 59.9 $\mathrm{mC})$ appeared to be higher than that of the females $(95.2 \pm 46.3$ $\mathrm{mC}$ ), but this difference was not statistically significant. Also, the IST was not statistically different between patients taking clozapine $(105.1 \pm 50.1 \mathrm{mC})$ and those not $(108.0 \pm 66.0 \mathrm{mC})$. The mean daily clozapine dose in 40 patients treated with clozapine was $262.5 \pm 126.3 \mathrm{mg}$ (range: $37.5-450 \mathrm{mg}$ ).

A multiple regression analysis with stepwise variable selection procedure suggested that age, gender and the dose per body weight $(\mathrm{mg} / \mathrm{kg})$ of sodium thiopental were the most relevant and parsimonious set of predictors (Table 2). Interesting$\mathrm{ly}$, if the unadjusted dose ( $\mathrm{mg}$ ) of sodium thiopental were substituted for the per-kilogram dosage, BMI and height emerged as other potentially relevant predictors. Therefore, it was inferred that per kilogram dosage of anesthetics, not the absolute dosage, was important in determining IST. The final re- gression model was highly significant $\left(\mathrm{F}_{[3,52]}=4.806, \mathrm{p}=0.005\right)$, the adjusted R-squared value was 0.172 , and each predictor (age, gender, and dose of sodium thiopental) was significant $(\mathrm{p}=0.05)$.

The relationship between IST and the set of relevant predictors (age, gender, and a dose of sodium thiopental) is depicted in Figure 2. For a visual representation, the dose of sodium thiopental was categorized into three non-overlapping ranges with equal numbers of patients. A positive relationship between age and IST was apparent in all subsets of the study patients, except for females receiving the middle range of the sodium thiopental dose (2.8-3.2 mg/kg) (Figure 2A). Additionally, higher IST values in males were clearly observed in those receiving the lower $(2.0-2.8 \mathrm{mg} / \mathrm{kg})$ and higher $(3.2-5.0 \mathrm{mg} / \mathrm{kg})$ ranges of sodium thiopental. The positive relationship between IST and sodium thiopental dose was more evident using another visual representation (Figure $2 \mathrm{~B}$ ) in which the dose of

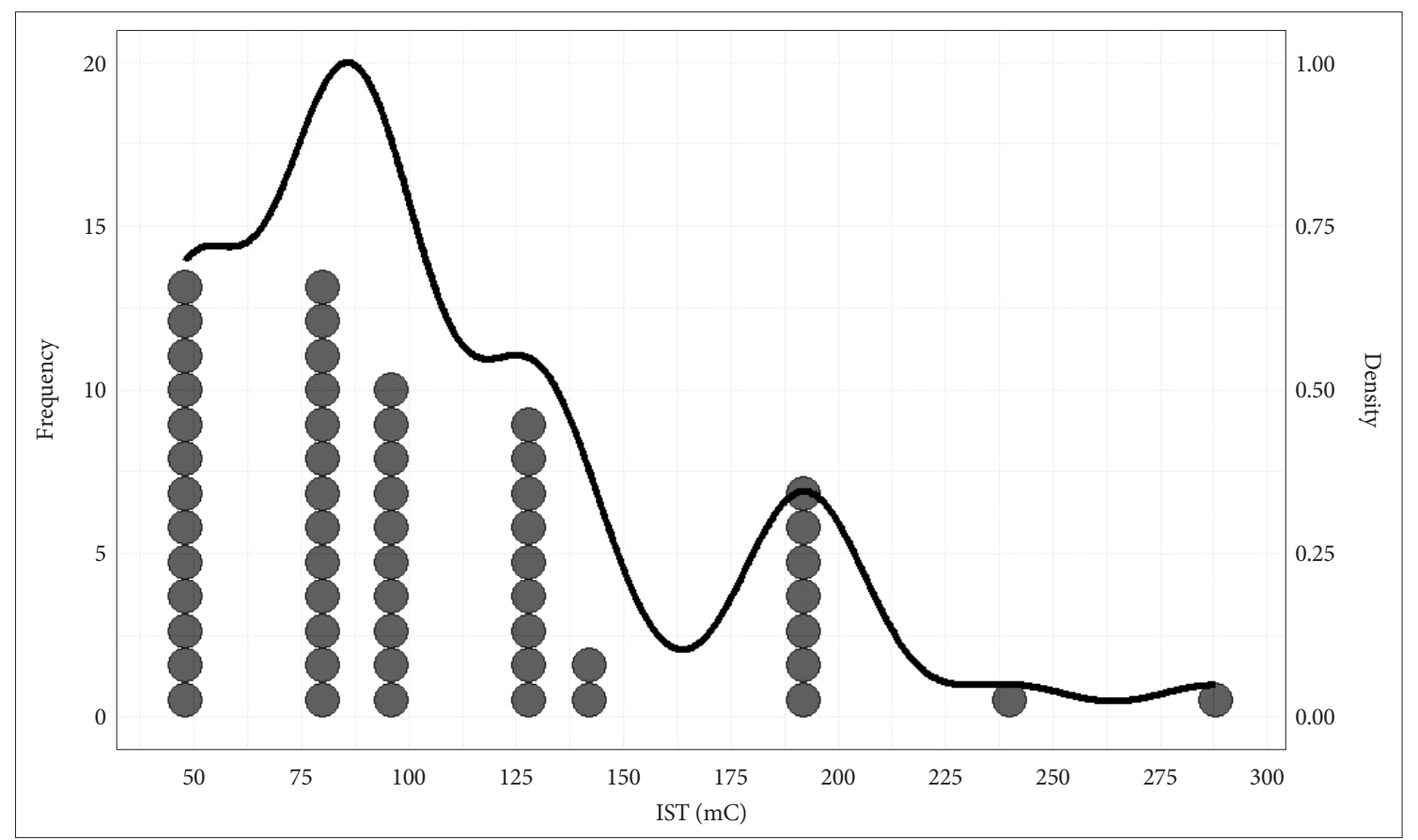

Figure 1. The distibution of IST after 1-millisecond pulse width BP-BL ECT. The IST values were estimated by empirical titration methods according to a predefined protocol. The subjects were 56 patients with schizophrenia and schizophreniform disorder. ECT was administered after anesthesia with sodium thiopental (gray dots are the actual cases and the thick black line is the estimated kernel density). IST: initial seizure threshold, BP-BL: brief-pulse bilateral, ECT: electrovulsive therapy.

Table 2. The final output of multiple regression analysis with IST as the dependent variable after the stepwise variable selection procedure

\begin{tabular}{|c|c|c|c|c|c|}
\hline Predictors* & $\beta$ estimate & Standardized $\beta$ & Standard error & $\mathrm{t}$-value & p-value \\
\hline Age & 1.63 & 0.354 & 0.59 & 2.76 & 0.008 \\
\hline Gender & -33.65 & -0.337 & 14.03 & -2.40 & 0.020 \\
\hline Thiopental dose per weight (mg/kg) & 27.35 & 0.291 & 11.53 & 2.37 & 0.021 \\
\hline Overall F-statistic: $4.81(3,52)$ & $\mathrm{p}=0.005$ & & $\mathrm{R}^{2}=0.217$ & \multicolumn{2}{|c|}{ Adjusted $\mathrm{R}^{2}=0.172$} \\
\hline
\end{tabular}

*these predictors were selected as the most parsimonious set of influential predictors for predicting IST. The most optimal model was chosen based on AIC. All predictors contained in the model significantly predicted IST values at p-values $<0.05$. The model explained $21.7 \%$ of the total variance. IST: initial seizure threshold, AIC: Akaike’s Information Criteria 
sodium thiopental had a clear effect on IST, except in male subjects older than 42 years of age. Visual inspections were indicative of a potential interaction among the relevant variables. In order to verify the consistency of the pairwise associations across different strata of other covariates, complex interaction models were evaluated. However, the interaction was not statistically significant in any of the models.

Other clinical variables related to the use of concurrent med-
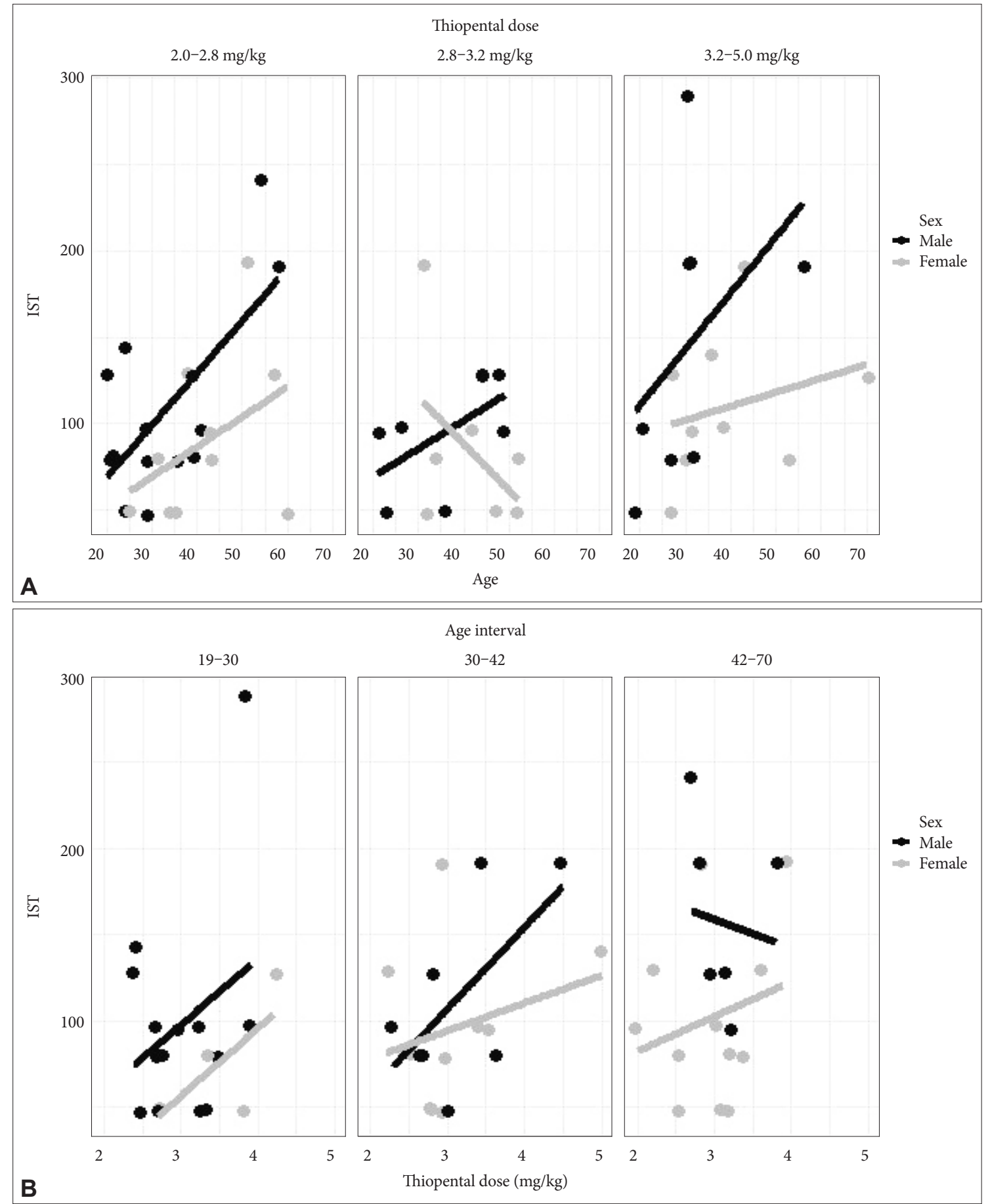

Figure 2. Relationships between IST and age, gender, and dose of sodium thiopental in patients who underwent BP-BL ECT after anesthesia with sodium thiopental. The overlaid lines represent the linear regression lines through respective subsets of study subjects. A: Relationship between IST and age stratified by gender and dose $(\mathrm{mg} / \mathrm{kg})$ of sodium thiopental using an arbitrary categorization of dose into three non-overlapping ranges with equal numbers of patients. B: Relationship between IST and dose $(\mathrm{mg} / \mathrm{kg})$ of sodium thiopental stratified by gender and age using an arbitrary categorization of age into three non-overlapping ranges with equal numbers of patients. IST: initial seizure threshold, BP-BL: brief-pulse bilateral, ECT: electrovulsive therapy. 
ications (clozapine, anticonvulsants, antidepressants, and benzodiazepines) were not significantly related to IST. Neither were they included in the final model nor any of the t-test for each variable revealed group difference.

\section{DISCUSSION}

This study explored the overall IST distribution in Korean patients with schizophrenia and schizoaffective disorder. The mean and median values of IST were $105.9 \mathrm{mC}$ and $96 \mathrm{mC}$ respectively. The distribution was highly right-skewed with about $80 \%$ of the study subjects below the IST value of $128 \mathrm{mC}$. The range was $48-288 \mathrm{mC}$ and the variability was a six-fold difference. The obtained IST was much higher than those reported from unilateral electrode placement. ${ }^{19,43}$

The largest studies so far which have explored IST values in representative samples of patients were done by Nitturkar et al. ${ }^{44}$ in India and by Petrides et al. ${ }^{10}$ in the United States. Both studies also employed the BP-BL ECT but comparing these studies with the present study involved a variety of problems. The subjects' diagnoses in the previous two studies were mostly mood disorders, and only a part of them included subjects with schizophrenia. Besides, the US study used methohexital as anesthetic agent thus making the comparison difficult. On the other hand, the Indian study used thiopentone as in the present study. Despite having many aspects in common with the present study, the IST value reported by Nitturkar et al. ${ }^{44}$ was higher (mean: $130.4 \mathrm{mC}$ ) than that in the present study (mean: $105.9 \mathrm{mC}$ ). They reported that IST values of $78.6 \%$ of the patients were equal or above $120 \mathrm{mC}$. The corresponding percentage in the present study was only $35.7 \%$. Petrides et al. ${ }^{10}$ also reported higher IST value (mean: $126.0 \mathrm{mC}$ ) and about $80 \%$ of patients were between 100 and $200 \mathrm{mC}$. The percentage belonging to this range in the present study was only $32 \%$.

This comparison gives the impression that the IST values from Korean subjects are rather low than from the American or Indian population. However, IST values reported in other small or medium-sized studies were in the lower or similar range as the present study irrespective of race or ethnicity (Table 3). Especially, the result from Chinese subjects was very close to our result. ${ }^{29,30}$ Above all, the only meta-analysis of IST conducted by van Waarde et $\mathrm{al}^{43}$ reported $111.6 \mathrm{mC}$ as the weighted mean of past studies, which roughly corresponded to the present study. Another Korean study employing the same BP-BL ECT reported the mean IST values between 32.89-45.23 Joules (in different conditions) which corresponded to 186.7$257.9 \mathrm{mC}$. ${ }^{24}$ The use of propofol as anesthetic agent might have caused the overall upward adjustment in this study. This range was even higher than those of Nitturka et al. $^{44}$ and Petrides et al. ${ }^{10}$ Taken together, even though there remained a question of how to reconcile the differences in subject composition and employed ECT methodology, the ethnic difference seemed not to be greater than the differences between individual studies.

This study replicated the well-known fact that IST values are significantly associated with age and gender. Older age and being male predict a higher IST., ${ }^{3,15,18,26}$ Although the contribution of age and gender was only modest, the close relationship among these variables is the basis of the classical dose recommendation established by Coffey et al. ${ }^{15}$ Meanwhile, previous notion that concomitant medications, such as, mood stabiliz-

Table 3. Previous studies of the IST distributions in bilateral electroconvulsive therapy

\begin{tabular}{|c|c|c|c|c|c|c|c|}
\hline Study & IST $($ Mean \pm SD) & Median & Range & Diagnosis & Anesthetics & Ethnicity & $\begin{array}{l}\text { Number of } \\
\text { participants }\end{array}$ \\
\hline Coffey et al. ${ }^{15}$ & $86.4 \pm 34.9$ & & & Affective disorder & Methohexital & American & 25 \\
\hline Scott et al..$^{20}$ & & 75 & $50-200$ & Depressive disorder & Methohexitone & European & 100 \\
\hline Chanpattana et al. ${ }^{28}$ & $91.3 \pm 31.2$ & & & Schizophrenia & Thiopental & Asian (Thai) & 93 \\
\hline Chung and Wong ${ }^{29}$ & $117.4 \pm 65.6$ & & $48-403$ & Mixed & Thiopentone & Asian (Chinese) & 54 \\
\hline Chung and Wong ${ }^{30}$ & $105.5 \pm 40.6$ & & $48-192$ & Mixed thiopentone & & Asian (Chinese) & 22 \\
\hline Chung $^{56}$ & 115.1 & & $48-403$ & Mixed & Thiopentone & Asian (Chinese) & 105 \\
\hline Petrides et al. ${ }^{10}$ & $126.0 \pm 69.7$ & 100 & $25-400$ & Depressive disorder & Methohexital & American & 402 \\
\hline van Waarde et al. ${ }^{43}$ & 111.6 & & & Mixed & Mixed & Meta-analysis & 1,116 \\
\hline Bennett et al..$^{55}$ & & 125 & $50-300$ & Depressive disorder & (Not informed) & European & 62 \\
\hline van Waarde et al. ${ }^{18}$ & $95.4 \pm 59.5$ & & & Depressive disorder & Etomidate & European & 28 \\
\hline Yashuda et al. ${ }^{23}$ & 136 & & $50.4-403$ & Major depressive disorder & Thiamylal & Asian (Japanese) & 54 \\
\hline Nitturkar et al. ${ }^{44}$ & $130.4 \pm 52.0$ & & & Mixed & Thiopentone & Asian (Indian) & 640 \\
\hline Chi et al. ${ }^{24}$ & $186.7 \pm 35.1$ & & & Mixed & Propofol & Asian (Korean) & 43 \\
\hline Present study & $105.9 \pm 54.5$ & 96 & $48-288$ & Schizophrenia & Thiopental & Asian (Korean) & 56 \\
\hline
\end{tabular}

SD: standard deviation, IST: initial seizure threshold 
ing agents, benzodiazepine, and antipsychotics (especially clozapine) may influence the seizure threshold was not verified in the present study. Quite a few preclinical studies have addressed the potential influence of psychotropic drugs on seizure physiology, but the actual ECT experience was inconsistent. ${ }^{45-47}$ Interaction among the psychotropic drugs themselves, complicated dose-response relationship, strong effect of anesthetic agent, or anticonvulsant effect of ECT itself may be some of the intricacies involved in these inconsistent results. ${ }^{18}$ Due to these intricacies and the small sample size of the present study, it would be premature to conclude that concomitant medications do not have any effects on IST.

Apart from this, another finding was the association between thiopental dose and IST. Traditionally, methohexital, etomidate, thiopental, and propofol have been used in ECT and there is no robust evidence to recommend a particular agent. ${ }^{48,49} \mathrm{An}$ ideal induction agent should have a short half-life and have no interference with seizure duration or threshold. However, these anesthetics exhibit dose-dependent anti- or pro-convulsant activity and thus may change the seizure threshold. ${ }^{49}$ Among these, propofol has the highest potential and thiopental/etomidate has the least potential. ${ }^{49,50}$ Although, the dose of anesthetic agent is rarely mentioned as a determinant of seizure threshold, a couple of authors stressed the importance of anesthetic dosage in seizure threshold. ${ }^{19,51}$ In the study by Boylan et al., ${ }^{16}$ the obtained multivariate regression model only contained age, sex and methohexital dose, which was the same conclusion as the present study. Despite the methodological difference using right unilateral electrode placement, Galvez et al. ${ }^{19}$ also observed that age, the kind and dose of anesthetic agent significantly predicted the IST value.

In an ECT center, an operation protocol is usually predefined according to the characteristics of the patient population and the ECT equipment. It dictates the employed ECT method, stimulus dose titration method, anesthesia-related procedures and policy on concomitant medication. In this case, estimating the IST distribution and finding the possible predictors of IST on that protocol would be important in several aspects. The Royal College of Psychiatrists recommends that the selection of the electrical dose should be individualized and contingent upon the IST value determined in the first session. ${ }^{52}$ They also pointed out that since IST cannot be reliably predicted based on demographic or clinical features, the empirical titration method is the preferred practice. ${ }^{53}$ However, since this method estimates IST by starting from the lowest intensity and then incrementally increasing the stimulus, repeated exposure to subthreshold electrical stimuli might be an unintended consequence. ${ }^{27}$ It may lead to cardiovascular complications, unwanted prolonged anesthesia, and more cognitive side effects. ${ }^{54}$
Thus, the balance between harm and benefit of the empirical titration partly depends on the location and range of the expected IST value distribution. Past authors have had very different views on the extent of the IST variability. Some authors reported as high as 40 -fold variation, ${ }^{3,11}$ while others suggested only 4-6 fold variability. ${ }^{13,16,30}$ The variability in the present study was 6-fold which is relatively narrow compared to previously known values. If the expected variability is narrow, the number of repeated trials would be small, and the risk of overexposure ignorable. However, in the opposite case, the number of unnecessary trials will increase beyond the allowed limit. Likewise, if the gap between the actual distribution of IST and the clinician's unwarranted expectation becomes wide, then the harmful effect of titration might exceed the potential benefit. In the latter two cases, efforts may have to be made to predict the IST value in advance before starting a titration procedure. Demographic and clinical variables associated with IST would be very useful in this situation even if the prediction is not precise.

The present study described an IST reference in Korean patients following a certain predefined protocol as an example. In order to successfully manage and to set up a procedure protocol of ECT, a preliminary determination of IST values stratified by sex, age and other variables may have to be defined based on past experience and accumulated data. In this case, the reference data described in the present study could be helpful in deciding whether the obtained IST values were within a comparable range and whether the similar variables could partly predict the individual IST values. Admittedly, the number of subjects in the present study was only modest for obtaining adequate generalizability and for properly investigating the determinants of IST with all their interactions. This may limit the value of the present findings. Undoubtedly, a universal reference which can be applied to every case is fundamentally unobtainable since every ECT center would deal with different patient populations and employ different ECT procedures. Minor changes in the implemented protocol may influence the overall distribution. Therefore, the data in the present study could only be used as a starting point to define each center's own reference guide suited to its own needs

In conclusion, the present study reported an IST value distribution in Korean patients and replicated the previous finding that age and gender were independent predictors of IST. Additionally, it demonstrated that the dose of sodium thiopental per body weight was another predictor of IST. The described IST distribution was comparable to previous studies and the empirical titration method seemed appropriate to deal with most of the cases. Taken together, these findings may be used as a reference for estimating the IST in patients with schizophrenia or schizoaffective disorder. However, it has to 
be mentioned that this result only applies to Korean patients who underwent 1-ms BP-BL ECT following sodium thiopental anesthesia. It may limit the generalizability of the study result.

\section{Acknowledgments}

This study was supported by research grants of Clinical Institute of Psychopharmacology, Dongguk University Medical Center and Whanin Pharm. Co. LTD.

\section{Conflicts of Interest}

Dr. Kim YS received grants, research support and honoraria from Janssen, Otsuka, and Dainippon Sumitomo. Dr. Chung IW received research and educational grants from Janssen, Otsuka, Hanmi, Mecta, and Whanin. Dr. Youn T received research and educational grants from Otsuka, Eisai, Hanmi, Lundbeck, and Mecta. The others have nothing to declare.

\section{Author Contributions}

Conceptualization: Yong Sik Kim, In Won Chung. Data curation: Young Wook Jeong, Jin Hyeok Jang. Formal analysis: Seong Hoon Jeong, Tak Youn. Funding acquisition: Yong Sik Kim, In Won Chung. Investigation: Yong Sik Kim, In Won Chung, Young Wook Jeong. Methodology: In Won Chung, Jin Hyeok Jang, Tak Youn. Project administration: In Won Chung, Tak Youn. Resources: In Won Chung, Yong Sik Kim. Software: Seong Hoon Jeong, Younsuk Lee, Jin Hyeok Jang. Supervision: Yong Sik Kim, Younsuk Lee. Validation: Seong Hoon Jeong, Younsuk Lee, Tak Youn. Visualization: Seong Hoon Jeong, In Won Chung. Writing-original draft: Seong Hoon Jeong, In Won Chung. Writing_review \& editing: Yong Sik Kim, Seong Hoon Jeong.

\section{ORCID iDs}

In Won Chung https://orcid.org/0000-0002-7852-7430

Seong Hoon Jeong https://orcid.org/0000-0002-7404-9056

\section{REFERENCES}

1. Kellner CH, Greenberg RM, Murrough JW, Bryson EO, Briggs MC, Pasculli RM. ECT in treatment-resistant depression. Am J Psychiatry 2012;169:1238-1244.

2. Pompili M, Lester D, Dominici G, Longo L, Marconi G, Forte A, et al. Indications for electroconvulsive treatment in schizophrenia: a systematic review. Schizophr Res 2013;146:1-9.

3. Sackeim H, Decina P, Prohovnik I, Malitz S. Seizure threshold in electroconvulsive therapy. Effects of sex, age, electrode placement, and number of treatments. Arch Gen Psychiatry 1987;44:355-360.

4. McCall WV, Reboussin DM, Weiner RD, Sackeim HA. Titrated moderately suprathreshold vs fixed high-dose right unilateral electroconvulsive therapy: acute antidepressant and cognitive effects. Arch Gen Psychiatry 2000;57:438-444.

5. Loo CK, Schweitzer I, Pratt C. Recent advances in optimizing electroconvulsive therapy. Aust N Z J Psychiatry 2006;40:632-638.

6. Abrams R. Electroconvulsive Therapy. Oxford: Oxford University Press; 2002.

7. McClintock SM, Choi J, Deng ZD, Appelbaum LG, Krystal AD, Lisanby $\mathrm{SH}$. Multifactorial determinants of the neurocognitive effects of electroconvulsive therapy. J ECT 2014;30:165-176.

8. Tiller JW, Ingram N. Seizure threshold determination for electroconvulsive therapy: stimulus dose titration versus age-based estimations. Aust N Z J Psychiatry 2006;40:188-192.

9. Ward WK, Lush P, Kelly M, Frost AD. A naturalistic comparison of two right unilateral electroconvulsive therapy dosing protocols: 2-3X seizure threshold versus fixed high-dose. Psychiatry Clin Neurosci 2006;60:429-433.
10. Petrides G, Braga RJ, Fink M, Mueller M, Knapp R, Husain M, et al. Seizure threshold in a large sample: implications for stimulus dosing strategies in bilateral electroconvulsive therapy: a report from CORE. J ECT 2009;25:232-237.

11. Sackeim HA, Prudic J, Devanand DP, Kiersky JE, Fitzsimons L, Moody BJ, et al. Effects of stimulus intensity and electrode placement on the efficacy and cognitive effects of electroconvulsive therapy. $\mathrm{N}$ Engl J Med 1993;328:839-846.

12. Enns M, Karvelas L. Electrical dose titration for electroconvulsive therapy: a comparison with dose prediction methods. Convuls Ther 1995; 11:86-93.

13. Sackeim HA, Devanand DP, Prudic J. Stimulus intensity, seizure threshold, and seizure duration: impact on the efficacy and safety of electroconvulsive therapy. Psychiatr Clin North Am 1991;14:803-843.

14. McCall WV, Shelp FE, Weiner RD, Austin S Norris J. Convulsive threshold differences in right unilateral and bilateral ECT. Biol Psychiatry 1993;34:606-611.

15. Coffey CE, Lucke J, Weiner RD, Krystal AD, Aque M. Seizure threshold in electroconvulsive therapy: I. Initial seizure threshold. Biol Psychiatry 1995;37:713-720.

16. Boylan LS, Haskett RF, Mulsant BH, Greenberg RM, Prudic J, Spicknall $\mathrm{K}$, et al. Determinants of seizure threshold in ECT: benzodiazepine use, anesthetic dosage, and other factors. J ECT 2000;16:3-18.

17. Bundy BD, Hewer W, Andres FJ, Gass P, Sartorius A. Influence of anesthetic drugs and concurrent psychiatric medication on seizure adequacy during electroconvulsive therapy. J Clin Psychiatry 2010;71:775777.

18. van Waarde JA, van Oudheusden LJ, Verwey B, Giltay EJ, van der Mast RC. Clinical predictors of seizure threshold in electroconvulsive therapy: a prospective study. Eur Arch Psychiatry Clin Neurosci 2013;263: 167-175.

19. Galvez V, Hadzi-Pavlovic D, Smith D, Loo CK. Predictors of seizure threshold in right unilateral ultrabrief electroconvulsive therapy: role of concomitant medications and anaesthesia used. Brain Stimul 2015;8: 486-492.

20. Scott AI, Dykes S. Initial seizure threshold in the clinical practice of bilateral electroconvulsive therapy in Edinburgh, Scotland. J ECT 1999;15:118-124.

21. Dawkins K, Ekstrom RD, Hill MA, Isaacs DL, Golden RN. Ethnicity and seizure threshold. Prog Neuropsychopharmacol Biol Psychiatry 2000;24:1289-1298.

22. Williams MD, Rummans T, Sampson S, Knapp R, Mueller M, Husain $\mathrm{MM}$, et al. Outcome of electroconvulsive therapy by race in the Consortium for Research on Electroconvulsive Therapy multisite study. J ECT 2008;24:117-121.

23. Yasuda K, Kobayashi K, Yamaguchi M, Tanaka K, Fujii T, Kitahara Y, et al. Seizure threshold and the half-age method in bilateral electroconvulsive therapy in Japanese patients. Psychiatry Clin Neurosci 2015; 69:49-54.

24. Chi SH, Jeong HG, Lee S, Oh SY, Kim SH. Effects of psychotropic drugs on seizure threshold during electroconvulsive therapy. Psychiatry Investig 2017;14:647-655.

25. Petrides G, Fink M. The "half-age" stimulation strategy for ECT dosing. Convuls Ther 1996;12:138-146.

26. Swartz CM, Michael N. Age-based seizure threshold determination. J ECT 2013;29:18-20.

27. Rosenman SJ. Electroconvulsive therapy stimulus titration: not all it seems. Aust N Z J Psychiatry 2018;52:410-414.

28. Chanpattana W, Buppanharun W, Raksakietisak S, McCall WV, Somchai Chakrabhand ML. Seizure threshold rise during electroconvulsive therapy in schizophrenic patients. Psychiatry Res 2000;96:31-40.

29. Chung KF, Wong SJ. Initial seizure threshold of bilateral electroconvulsive therapy in Chinese. J ECT 2001;17:254-258.

30. Chung KF, Wong SJ. Stimulus dose titration for electroconvulsive therapy. Psychiatry Clin Neurosci 2001;55:105-110. 
31. Lee MR, Ahn JH, Lee C, Han OS, Kim CY. Guidelines for the adequate initial electric stimuli in bilateral ECT using MECTA device. Korean J Psychopharmacol 1999;10:138-142.

32. Abrams R. Stimulus titration and ECT dosing. J ECT 2002;18:3-9; discussion $14-15$

33. Joo SW, Joo YH, Kim CY, Lee JS. Effects of stimulus parameters on motor seizure duration in electroconvulsive therapy. Neuropsychiatr Dis Treat 2017;13:1427-1434.

34. Bersani G, Iannitelli A, Caredda M, Bersani FS, Orsi P, Pacitti F. Seizure threshold variations in ECT-treated chronic patients with schizophrenia: a brief report. Riv Psichiatr 2014;49:84-88.

35. Pawelczyk T, Kolodziej-Kowalska E, Pawelczyk A, Rabe-Jablonska J. Effectiveness and clinical predictors of response to combined ECT and antipsychotic therapy in patients with treatment-resistant schizophrenia and dominant negative symptoms. Psychiatry Res 2014;220:175-180.

36. Lally J, Tully J, Robertson D, Stubbs B, Gaughran F, MacCabe JH. Augmentation of clozapine with electroconvulsive therapy in treatment resistant schizophrenia: A systematic review and meta-analysis. Schizophr Res 2016;171:215-224.

37. Kim HS, Kim SH, Lee NY, Youn T, Lee JH, Chung S, et al. Effectiveness of electroconvulsive therapy augmentation on clozapine-resistant schizophrenia. Psychiatry Investig 2017;14:58-62.

38. Bueno CR, Rosa MO, Rumi DO, Ribeiro RB, Rosa MA. Cardiovascular safety of the method of limits titration procedure for electroconvulsive therapy dosing: a retrospective study. Brain Stimul 2011;4:43-45.

39. Mizen L, Morton C, Scott A. The cardiovascular safety of the empirical measurement of the seizure threshold in electroconvulsive therapy. BJPsych Bull 2015;39:14-18.

40. Coffey CE, Lucke J, Weiner RD, Krystal AD, Aque M. Seizure threshold in electroconvulsive therapy (ECT) II. The anticonvulsant effect of ECT. Biol Psychiatry 1995;37:777-788.

41. Rattehalli RD, Thirthalli J, Rawat V, Gangadhar BN, Adams CE. Measuring electroencephalographic seizure adequacy during electroconvulsive therapy: a comparison of 2 definitions. J ECT 2009;25:243-245.

42. R Core Team. R: A Language and Environment for Statistical Computing. https://www.R-project.org, Vienna, Austria, 2018.

43. van Waarde JA, Verwey B, van der Mast RC. Meta-analysis of initial seizure thresholds in electroconvulsive therapy. Eur Arch Psychiatry Clin Neurosci 2009;259:467-474.

44. Nitturkar AR, Sinha P, Bagewadi VI, Thirthalli J. Effect of age and anticonvulsants on seizure threshold during bilateral electroconvulsive ther- apy with brief-pulse stimulus: A chart-based analysis. Indian J Psychiatry 2016;58:190-197.

45. Galvez V, Loo CK, Alonzo A, Cerrillo E, Menchon JM, Crespo JM, et al. Do benzodiazepines moderate the effectiveness of bitemporal electroconvulsive therapy in major depression? J Affect Disord 2013;150: 686-690.

46. Tang VM, Pasricha AN, Blumberger DM, Voineskos D, Pasricha S, Mulsant $\mathrm{BH}$, et al. Should benzodiazepines and anticonvulsants be used during electroconvulsive therapy?: A case study and literature review. J ECT 2017;33:237-242.

47. Rakesh G, Thirthalli J, Kumar CN, Muralidharan K, Phutane VH, Gangadhar BN. Concomitant anticonvulsants with bitemporal electroconvulsive therapy: a randomized controlled trial with clinical and neurobiological application. J ECT 2017;33:16-21.

48. Hoyer C, Kranaster L, Janke C, Sartorius A. Impact of the anesthetic agents ketamine, etomidate, thiopental, and propofol on seizure parameters and seizure quality in electroconvulsive therapy: a retrospective study. Eur Arch Psychiatry Clin Neurosci 2014;264:255-261.

49. Kadiyala PK, Kadiyala LD. Anaesthesia for electroconvulsive therapy: an overview with an update on its role in potentiating electroconvulsive therapy. Indian J Anaesth 2017;61:373-380.

50. Zahavi GS, Dannon P. Comparison of anesthetics in electroconvulsive therapy: an effective treatment with the use of propofol, etomidate, and thiopental. Neuropsychiatr Dis Treat 2014;10:383-389.

51. Anand S, Thirthalli J, Gupta A, Kongasseri S, Tharayil BS, Keshavan $\mathrm{VH}$, et al. Anesthesia during electroconvulsive therapy: importance of dosage. J ECT 2010;26:145.

52. The ECT Handbook (Third Edition). London: RCPsych Publications; 2013.

53. Lemasson M, Rochette L, Galvao F, Poulet E, Lacroix A, Lecompte M, et al. Pertinence of titration and age-based dosing methods for Eelectroconvulsive therapy: an international retrospective multicenter study. J ECT 2018;34:220-226.

54. Wells DG, Zelcer J, Treadrae C. ECT-induced asystole from a sub-convulsive shock. Anaesth Intensive Care 1988;16:368-371.

55. Bennett DM, Perrin JS, Currie J, Blacklaw L, Kuriakose J, Rao A, et al. A comparison of ECT dosing methods using a clinical sample. J Affect Disord 2012;141:222-226.

56. Chung KF. Determinants of seizure threshold of electroconvulsive therapy in Chinese. J ECT 2006;22:100-102. 